Case Report

\title{
Unicameral Bone Cyst in the Proximal Humerus with Secondary Infection in an 18-Month-Old Foal
}

\author{
Maria C. Fugazzola, Christoph Klaus, and Christoph Lischer \\ The Department of Veterinary Clinical Sciences, Unit of Equine Medicine and Surgery, University of Teramo (Fugazzola); \\ V.le Crispi-Loc. Cartecchio, 64100 Teramo and Klinik für Pferde, Free University of Berlin (Klaus and Lischer) Oertzenweg 19b, \\ 14163 Berlin, Germany \\ Correspondence should be addressed to Maria C. Fugazzola; maria.fugazzola@gmail.com
}

Received 22 May 2014; Revised 7 July 2014; Accepted 8 July 2014; Published 22 July 2014

Academic Editor: Jeffrey Lakritz

Copyright (c) 2014 Maria C. Fugazzola et al. This is an open access article distributed under the Creative Commons Attribution License, which permits unrestricted use, distribution, and reproduction in any medium, provided the original work is properly cited.

\begin{abstract}
An 18-month-old warmblood filly was $4 / 5$ lame on the front right limb at referral and showed severe swelling of the right shoulder region and pain during manipulation of the shoulder region. Radiography revealed a roundish $5 \times 7 \mathrm{~cm}$ radiolucent area with defined borders within the greater tubercle and the presence of a fracture of the lateral tubercle associated with the cyst. Cellular blood count was $27,500 \mathrm{WBC} / \mu \mathrm{L}$ and serum biochemical analyses revealed fibrinogen of $855 \mathrm{mg} / \mathrm{dL}$. The fractured bone was removed surgically; the cyst debrided and filled with autologous cancellous bone graft. Three and five weeks after surgery the filly was reoperated on because of an osseous sequestrum and a periostal defect on the distal dorsolateral aspect of the pastern of the right hind limb and a septic synovitis of the DFTS of the left hind limb. Fifteen month after surgery the filly was not lame and was introduced to training. Unicameral bone cysts (UBC) are well described lesions, often associated to pathologic fracture in the proximal humerus of children but, until present, no scientific report exists of UBC in the foal. The prompt surgical management had a favorable outcome.
\end{abstract}

\section{Case Details}

An 18-month-old warmblood filly which was kept on pasture with other foals was referred to the clinic for horses of the Free University of Berlin because of sudden onset of severe lameness of the right front limb and a swelling in the shoulder region.

At presentation the horse was 4/5 lame (AAEP scale) on the front right limb showing a severe firm swelling of the shoulder region. Palpation revealed an increased skin temperature and elicited a painful response. All vital parameters were within normal range.

\section{Diagnostic Imaging}

A lateromedial and a cranio $45^{\circ}$ medial-caudolateral oblique view of the scapulohumeral joint and a cranioproximalcraniodistal oblique view of the humeral tubercles revealed a roundish $5 \times 7 \mathrm{~cm}$ radiolucent area with defined, unreactive borders within the greater tubercle; the lesion was visible in all three projections. A $4 \mathrm{~cm}$ long partly detached bony fragment lateral to the radiolucent area could be detected in the cranioproximal-craniodistal oblique projection (Figure 1).

The epiphyseal growth plate of the proximal humerus was visible distal to the greater tubercle in the lateromedial projection at the level of the radiolucent area (Figure 2).

The ultrasonographic examination confirmed effusion of the bicipital bursa with no evidence of pathological changes of the bicipital tendon. Furthermore, a fracture associated with the insertion of the infraspinatus tendon was diagnosed in the dorsolateral aspect of the proximal humerus (Figure 3 ).

Fluid retrieved from the bicipital bursa had a brown discolored appearance and contained 9600 nucleated cells $/ \mu \mathrm{L}$ and $5 \mathrm{gr} / \mathrm{dL}$ proteins (reference values: $<1000$ cells $/ \mu \mathrm{L}$ and $0,5-1 \mathrm{~g} / \mathrm{dL}$ total protein). Cellular blood count was $27,500 \mathrm{WBC} / \mu \mathrm{L}$ and serum biochemical analyses 


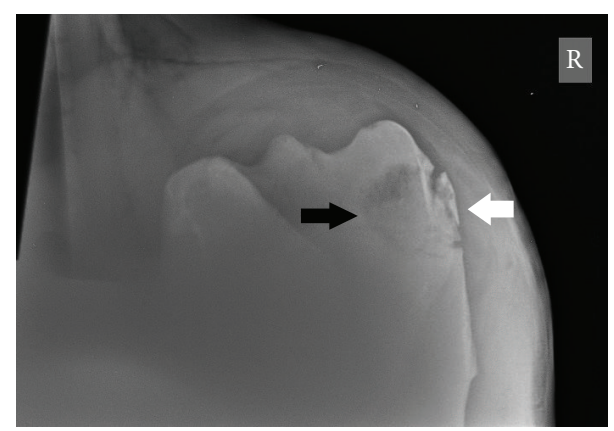

FIGURE 1: Cranioproximal-craniodistal oblique projection on the humeral tubercles: a $4 \mathrm{~cm}$ long partly detached bony fragment (white arrow) lateral to the radiolucent area (black arrow) is visible.

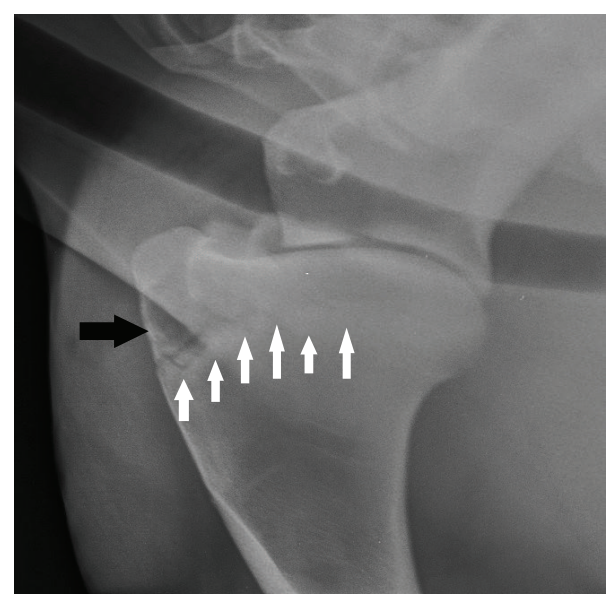

FIGURE 2: Lateromedial projection of the humerus: the epiphyseal growth plate of the proximal humerus (white arrows) is visible distal to the greater tubercle at the level of the radiolucent area (black arrow).

revealed $855 \mathrm{mg} / \mathrm{dL}$ of fibrinogen (reference values: 4,000$12,000 \mathrm{WBC} / \mu \mathrm{L}$ and $<300 \mathrm{mg} / \mathrm{dL}$ fibrinogen). All other CBC and biochemical parameters were within reference range.

The severe lameness, the probably septic bursitis of the intertubercular bursa, and the presence of the fracture close to the bone cyst determined the decision to treat the case surgically.

Under general anesthesia the filly was placed in lateral recumbency with the affected limb uppermost. The bicipital bursa was flushed during bursoscopy and small amounts of fibrin pannus were found adherent to the bursal wall, which was partially torn on its lateral aspect (Figure 4). No cartilaginous lesions could be detected in the intertubercular grooves.

A $15 \mathrm{~cm}$ long incision was performed in a proximodistal direction over the caudal eminence of the lateral tubercle, slightly distal to the infraspinatus tendon insertion. Dissection was performed bluntly until bone was exposed. The fractured bone was found in close proximity to the infraspinatus tendons attachment to the lateral tubercle and was removed. A cystic lesion of the tubercle became visible underlying the fragment, containing yellowish discolored fluid and fibrous

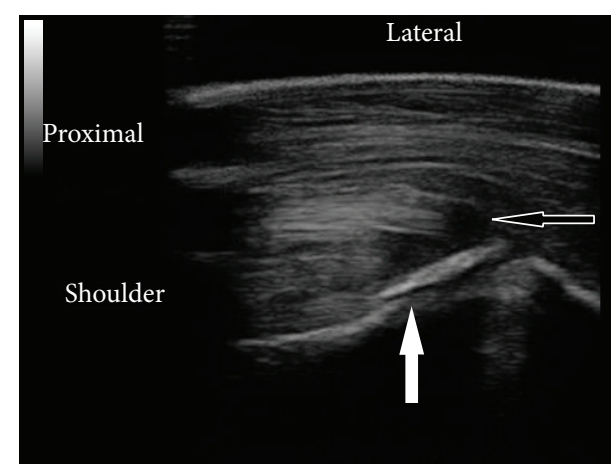

FIGURE 3: Ultrasound image of the lateral aspect of the greater tubercle of the humerus: a bony fragment (white arrow) associated with the insertion of the infraspinatus tendon (white empty arrow) on the distal aspect of the greater tubercle is visible.

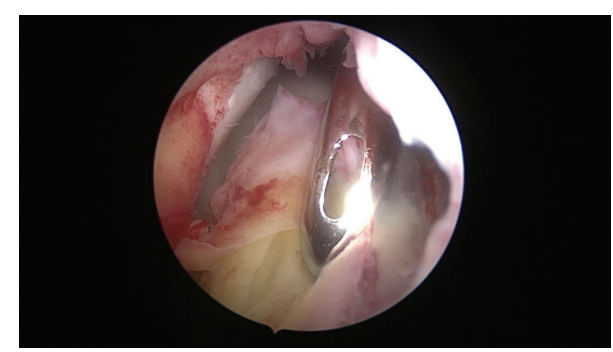

FIgURE 4: Bursoscopic image of the bicipital bursa: small amounts of fibrin pannus were found adherent to the bursal wall.

material that was evacuated and sent for histopathologic and microbiologic examination. After thorough debridement (Figure 5) and flushing with a disinfectant the bony defect was packed with autologous cancellous bone graft previously harvested from the ipsilateral tuber coxae.

Surgical closure was performed in 3 layers and the site was protected with a stent bandage until the day after surgery. Recovery from anesthesia was uneventful and an antibiotic therapy with ceftiofur sodium at $2,2 \mathrm{mg} / \mathrm{kg}$ (IM, q24h) was administered for 5 days postoperatively and then shifted to sulfonamide $25 \mathrm{mg} / \mathrm{kg}$ and trimethoprim $5 \mathrm{mg} / \mathrm{kg}$ (PO q12h) for 5 additional days. Anti-inflammatory medication consisted of phenylbutazone $2,2 \mathrm{mg} / \mathrm{kg}$ (PO q12h) that was discontinued when clinical symptoms had ceased.

$\mathrm{CBC}$ and plasma fibrinogen values were monitored every second day until discharge and showed a steady decrease with return to reference range by day 12 after surgery.

Histopathology of the cystic content revealed purulentnecrotic trabecular bone with fibrotic lining. Microbial culture revealed a severe gram positive Staphylococcus (Sp. S. aureus) infection with sensitivity among others to trimethoprim sulfonamide and ceftiofur.

Within two weeks the swelling of the shoulder had almost resolved completely and the filly was not lame at walk in the right front but showed a mild lameness on the hind right limb. Radiographically the cyst of the humerus showed a sclerotic rim (Figure 6). The hind right pastern region showed a warm, 


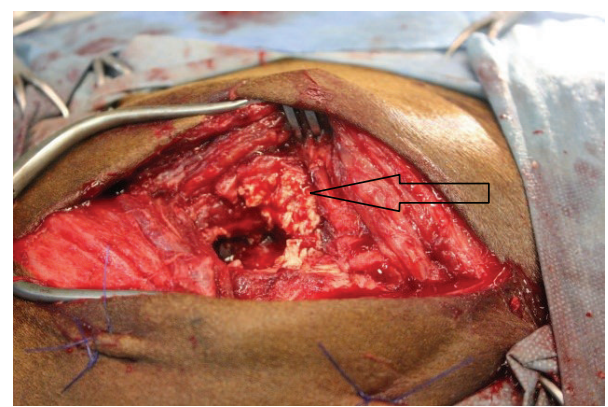

Figure 5: View of the bone cyst opening after debridement: proximal to the right and distal to the left. The debrided infraspinatus fibres (black arrow) are visible dorsal to the cyst opening.

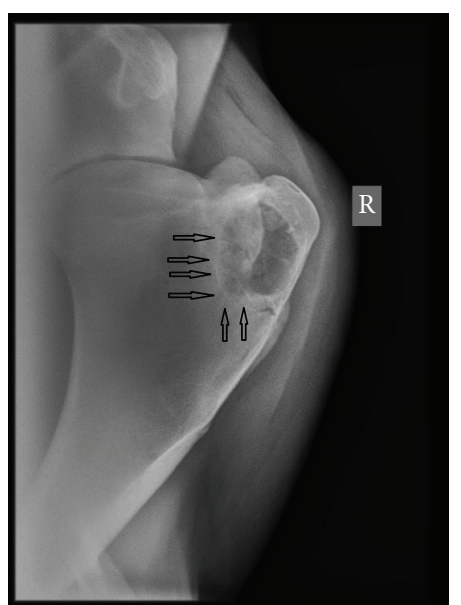

FIGURE 6: Cranial $45^{\circ}$ medial-caudolateral view of the humerus two weeks after surgery: the radiolucent area shows a sclerotic rim (black arrows).

painful swelling and the hind left digital flexor tendon sheath was mildly distended.

The dorsomedial-plantarolateral radiographic view of the right hind pastern showed osseous modelling of the periosteal surface of the distal dorsolateral aspect of the proximal phalanx, which could be confirmed by ultrasound. The proximal interphalangeal joint was not distended and synovial fluid could not be retrieved. Due to the strong suspect of a septic process spreading to the pastern, regional limb perfusion with $2 \mathrm{gr}$ of amikacin was performed twice in the following 4 days. Oral antibiotic administration of sulfonamide $25 \mathrm{mg} / \mathrm{kg}$ and trimethoprim $5 \mathrm{mg} / \mathrm{kg}$, q12h was resumed.

As after two weeks of antibiotic therapy no clinical improvement of the condition could be observed, diagnostic imaging of the right hind pastern was repeated showing progression of the periosteal reaction, in addition to the presence of a sequestrum and an osteolytic defect on the distal dorsolateral aspect of the proximal phalanx. Based on the assumption of an osteomyelitic process, the decision was made to remove the sequestrum and debride the site under general anesthesia. Due to the close proximity to the proximal interphalangeal joint a CT scan of the region was performed preceding the surgery (Figures $7(\mathrm{a})$ and $7(\mathrm{~b})$ ), in order to allow a precise localization and surgical approach. Before the skin incision was performed, the synovial fluid from the still notably distended hind left DFTS was analyzed. Values at this point were within reference ranges (cell number: 700 cells $/ \mu \mathrm{L}$, protein: $0,8 \mathrm{gr} / \mathrm{dL}$ ). A skin incision was made at the area of the sequestrum, which could be located and removed. An irregular osseous defect $1 \mathrm{~cm}$ long was debrided and flushed thoroughly. The proximal interphalangeal joint was opened during the procedure, since the joint capsule had its proximal attachment to the proximal phalanx in correspondence of the osteolytic site. Regional limb perfusion with 2 gr of amikacin was repeated during surgery and two days after surgery.

Histopathologic examination of parts of proximal interphalangeal joint capsule of the hind right showed a subacute capsulitis and synovitis with predominant lymphocytic and less neutrophilic infiltration; the cancellous bone that was removed through debridement of the osteomyelitic site on the distal aspect of the proximal phalanx showed only minimal lymphocytic infarction. Culture revealed colonies of Staphylococcus aureus two days later.

To identify a potential primary source of infection, ultrasonography of the heart, the lung, and the abdomen was performed with no relevant findings.

Six weeks after admission, the filly became lame on the left hind limb (grade 2/5); the distention of the DFTS in this limb had not regressed. Radiographic views of the left hind pastern showed no detectable abnormalities and synoviocentesis revealed a septic synovitis (protein $3 \mathrm{~g} / \mathrm{dL}, 17400$ cells/ $\mu \mathrm{L}$ with $92 \%$ of neutrophils). Flushing of the tendon sheath was performed under general anesthesia. Oral administration of sulfonamide $25 \mathrm{mg} / \mathrm{kg}$ and trimethoprim $5 \mathrm{mg} / \mathrm{kg}$, q12h was continued until 5 days after the tenovaginoscopy. Ten days after flushing the tendon sheath the lameness of the hind left had resolved.

Two months after admission to the clinic, the filly was discharged with grade 1/5 lameness on the right hind limb.

A clinical and radiographic follow-up examination was performed 6 and 15 months after admission to the clinic. The mare was not lame at trot. Radiographically the bone defect of the greater tubercle of the humerus showed radiopaque content within a sclerotic rim (Figure 8). The periostal defect of the hind right pastern showed an irregular dorsolateral osseous profile but no radiolucencies were visible any more. The mare started training successfully two months later.

\section{Discussion}

In pediatric medicine UBC is a single-chambered lesion that occurs mostly in the proximal humerus of skeletally immature individuals [1]. When such cysts are immediately adjacent to a growth plate, they are referred to as active cysts, whilst when they are distant from the growth plate they are considered to be latent cysts [1]. It is estimated that approximately $75 \%$ of children with unicameral bone cysts present pathological fractures involving the cyst location due to weakening of the bone [2]. In our case location of the cyst within the proximal aspect of the humerus, skeletal immaturity, and fracture involving the cyst match presentation 


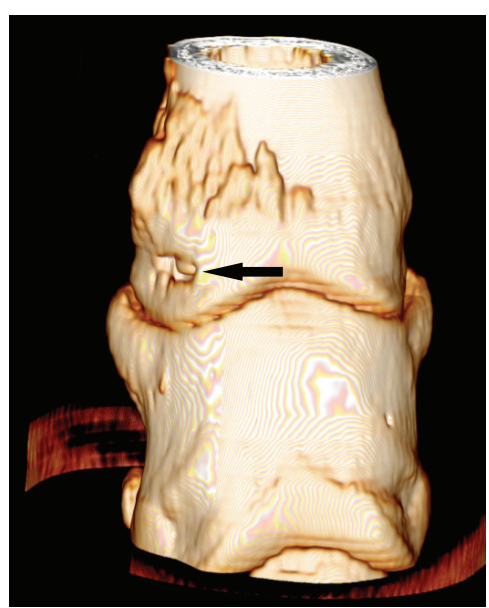

(a)

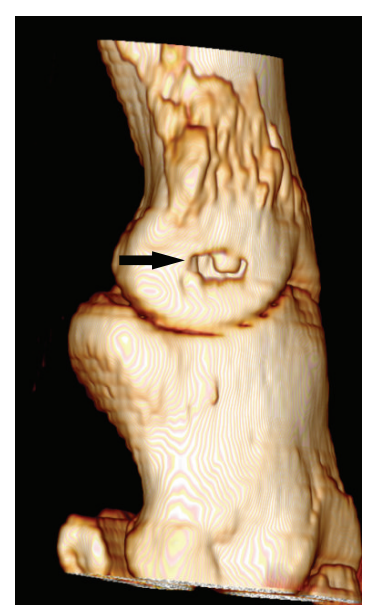

(b)

Figure 7: (a) and (b) 3D reconstruction of a CT scan of the hind right proximal interphalangeal joint area from a dorsal and lateral view. The osteolytic defect and the sequestrum (arrow) on the distal dorsolateral aspect of the proximal phalanx are visible.

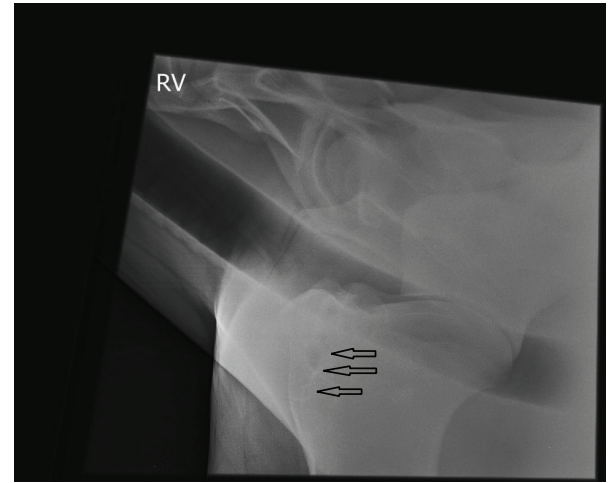

FIGURE 8: Lateromedial projection of the humerus 15 months after surgery: the bone defect of the humeral greater tubercle shows radiopaque content within a sclerotic rim.

criteria of a UBC. The characteristic radiographic appearance with absence of sclerotic rim and the involvement of the proximal growth-plate of the humerus represent a further typical feature which was evident also in the filly. Most cysts in pediatric medicine are an incidental finding when radiographs are taken for other reasons, proving that unless fractured they may be asymptomatic $[1,3]$. The symptoms of UBC are most often brought on by trauma [2]. In the present case the filly was kept with other yearling on pasture and was referred to be moderately lame from one day to the other. The most probably explanation for this would be a traumatic event that caused the humeral tubercle to fracture in the weakened location. During this trauma an undetected penetrating wound could then explain the infection with Staphylococcus aureus and explain the rapid worsening of the clinical symptoms, including swelling of the bicipital bursa in the following days preceding referral. The secondary spreading from the cyst to two distant sites would consequently be hematogenous. Alternatively the presence of septicemia, previously to the trauma, could have caused an infection of the cyst and a consequent hematogenous spreading to the hind right pastern and the hind left DFTS. UBC are fluid filled chambers with a fibrous membrane [2]. The infective process of the cyst explains the more extensive quantity of fibrous and purulent material found additionally to the fluid content in our case. Staphylococcus aureus represents a major cutaneous saprophyte in the horse enforcing the hypothesis of the infection of the preexisting bone cyst coming through undetected cutaneous trauma. The anatomic proximity to the epiphyseal growth plate of the humerus and of the greater tubercle which ossify after 24 months of age [4] could explain also the subsequent outbreak involving the right hind pastern bone and the DFTS of the hind left through a haematogenous pathway.

The humeral tubercles are an uncommon localization for osseous cyst-like lesions and only two case reports [5, 6] and one 5-case-series [7] have been reported in equine medicine. In one case the lesion was localized within the lateral tubercle of the humerus whilst in the others it was in the intermediate tubercle. The bicipital bursa was involved in 5 cases and in none of the cases a fracture of the tubercles could be detected. Follow-up was available in seven cases, of which one was submitted to euthanasia due to poor outcome [6] whilst all the horses of the 5-case-series underwent successful conservative therapy [7]; the last case was treated with surgical debridement of the cyst [5].

Unlike the present report, in the aforementioned cases no further complications arose, with resolution of the primary lesion after conservative (5 cases) or surgical (1 case) management. The infection of the cyst in our case most likely was the cause for the subsequent hematogenous spreading to the pastern bone and DFTS. The presented case also differs regarding the age of the horses. In the reported cases horses were of mean age of $10(\mathrm{SD} \pm 4$ ) years whilst our filly was 18 months old. In the 5-case series, altered biomechanics of the shoulder, as a result of trauma, lameness in other sites of the limb, or bicipital bursitis was suggested to be responsible for abnormal fibrocartilage wear patterns [7]. 
The pathogenesis was suggested to be microtrauma of the osteochondral surface leading to osseous cyst-like lesions through breakthrough of synovial fluid into the defect by hydraulic action $[8,9]$. The horses involved had a mean age of 10 years and were active in training; our filly was 18 months old and not broken. It is therefore unlikely that the lesion resulted from altered biomechanics.

Another possible differential diagnosis besides a UBC could be a primary bone infection, which subsequently caused the cyst to develop. Such lesions include a bone abscess, known as Brodie's Abscess in human literature and described in the equine proximal third metatarsal bone [10], first phalanx [11], lateral epicondyle of the humerus [12], and proximal tibia [13]. These lesions are usually confined to the diaphysis or metaphysis of long bones and are characterized by presence of a sclerotic rim [14]. Location of our lesion and absence of bone reaction of the cysts borders make this pathogenesis remote.

A primary osteomyelitis due to septicemia could have also been the cause of cyst development, although it is unlikely that such process would cause lameness in such an acute manner as in our case. In order for the infectious process to reach such extension within the bone a longer time span would have been necessary. Furthermore Staphylococcus aureus is a commonly isolated bacterium in human pediatric and adult horses' osteomyelitis $[15,16]$, but it is rare in foals [17]. The spreading of a primary bone infection to distant sites in young horses has been described. In a report of multiple bone infection in foals, Bennett et al. [18] retrieved Staphylococcus aureus always in proximity to physeal growth plates. As in our case, the multiple bone infection was assumed to spread by hematogenous pathway, either through septicaemia or by dissemination of infected emboli (septic emboli). There was involvement of other sites which were not suspected on initial clinical examination similar to our case. Although ossification of the physeal growth plate in the distal pastern bone occurs within the first month of life, the lesion in our case was in exact correspondence of where it once had been, whilst the proximal humeral growth plate was still open and was therefore probably the primary portal of the infection to the bloodstream.

A further pathogenesis could have been an overwhelming septicemia that seeded to the shoulder, the proximal phalanx of the right hind limb, and the DFTS of the left hind limb. In this case the infection of the cyst after the trauma would have been hematogenous.

The surgical removal of the fractured bone and the transosseous evacuation of the cystic content resolved the humeral lesion by decreasing intraosseous pressure, removing the cystic lining, and reducing inflammatory mediator release into the bursa as described by von Rechenberg et al. [19]. Similar procedure is nowadays the treatment of choice for unicameral bone cysts [20].

Plasma fibrinogen concentration has been recently shown to be a useful indicator of physeal or epiphyseal osteomyelitis in foals [21]. Our outcome is in line with these results and constant measurement of plasma fibrinogen throughout the hospitalization reflected the constant decrease along with clinical improvement of the condition after surgical treatment.

The present case reports for the first time the presence of a unicameral bone cyst in an 18-month-old foal. The favorable outcome was similar to that described in pediatric orthopedics [20].

\section{Conflict of Interests}

The authors declare that there is no conflict of interests regarding the publication of this paper.

\section{References}

[1] R. M. Wilkins, "Unicameral bone cysts," The Journal of the American Academy of Orthopaedic Surgeons, vol. 8, no. 4, pp. 217-224, 2000.

[2] J. H. Beaty and J. R. Kasser, "Pathologic fractures associated with tumors and unique conditions of the musculoskeletal system," in Rockwood \& Wilkins' Fractures in Children, J. P. Dormans and J. M. Flynn, Eds., pp. 136-137, Lippincott Williams \& Wilkins, 6th edition, 2006.

[3] J. Cohen, "Etiology of simple bone cyst," Journal of Bone and Joint Surgery. American Volume, vol. 52, no. 7, pp. 1493-1497, 1970.

[4] S. J. Dyson, "The elbow, brachium and shoulder," in Diagnosis and Management of the Lameness in the Horse, M. W. Ross and S. J. Dyson, Eds., p. 456, Elsevier, 2nd edition, 2011.

[5] C. E. Arnold, M. K. Chaffin, C. M. Honnas, M. A. Walker, and W. K. Heite, "Diagnosis and surgical management of a subchondral bone cyst within the intermediate tubercle of the humerus in a horse," Equine Veterinary Education, vol. 20, no. 6, pp. 310-315, 2008.

[6] P. H. L. Ramzan, "Osseous cyst-like lesion of the intermediate humeral tubercle of a horse," The Veterinary Record, vol. 154, no. 17, pp. 534-536, 2004.

[7] D. Little, W. R. Redding, and M. P. Gerard, "Osseous cyst-like lesions of the lateral intertubercular groove of the proximal humerus: a report of 5 cases," Equine Veterinary Education, vol. 21, no. 2, pp. 60-66, 2009.

[8] L. B. Jeffcott, S. E. Kold, and F. Melsen, "Aspects of the pathology of stifle bone cysts in the horse," Equine veterinary Journal, vol. 15, no. 4, pp. 304-311, 1983.

[9] F. Verschooten and A. de Moor, "Subchondral cystic and related lesions affecting the equine pedal bone and stifle," Equine Veterinary Journal, vol. 14, no. 1, pp. 47-54, 1982.

[10] J. M. White, K. J. Hughes, C. Scruton, J. Morris, A. W. Philbey, and C. J. Lischer, "Intramedullary abscess of the proximal third metatarsal bone in a 4-year-old Thoroughbred horse," Equine Veterinary Education, vol. 19, no. 6, pp. 291-296, 2007.

[11] J. A. Hanson, H. J. Seeherman, C. A. Kirker-Head, and M. W. O'Callaghan, "The role of computed tomography in evaluation of subchondral osseous lesions in seven horses with chronic synovitis," Equine Veterinary Journal, vol. 28, no. 6, pp. 480-488, 1996.

[12] M. J. Huber and G. R. Grisel, "Abscess on the lateral epicondyle of the humerus as a cause of lameness in a horse," Journal of the American Veterinary Medical Association, vol. 211, no. 12, pp. 1558-1561, 1997.

[13] B. D. Young, D. A. Hendrickson, and R. D. Park, "What is your diagnosis? Mixed lytic-proliferative lesion in the left tibia," 
Journal of the American Veterinary Medical Association, vol. 221, no. 9, pp. 1251-1252, 2002.

[14] T. D. Lopes, W. R. Reinus, and A. J. Wilson, "Quantitative analysis of the plain radiographic appearance of Brodie's abscess," Investigative Radiology, vol. 32, no. 1, pp. 51-58, 1997.

[15] J. Dartnell, M. Ramachandran, and M. Katchburian, "Haematogenous acute and subacute paediatric osteomyelitis," Journal of Bone and Joint Surgery B, vol. 94, no. 5, pp. 584-595, 2012.

[16] G. W. Trotter, "Osteomyelitis," in Equine Fracture Repair, A. J. Nixon, Ed., p. 359, Saunders, Philadelphia, Pa, USA, 1st edition, 1996.

[17] R. M. Moore, R. K. Schneider, J. Kowalski, L. R. Bramlage, L. M. Mecklenburg, and C. W. Kohn, "Antimicrobial susceptibility of bacterial isolates from 233 horses with musculoskeletal infection during 1979-1989," Equine Veterinary Journal, vol. 24, no. 6, pp. 450-456, 1992.

[18] D. Bennett, "Pathological features of multiple bone infection in the foal.," Veterinary Record, vol. 103, no. 22, pp. 482-485, 1978.

[19] B. von Rechenberg, H. Guenther, C. W. McIlwraith et al., "Fibrous tissue of subchondral cystic lesions in horses produce local mediators and neutral metalloproteinases and cause bone resorption in vitro," Veterinary Surgery, vol. 29, no. 5, pp. 420429, 2000.

[20] F. Canavese, J. G. Wright, W. G. Cole, and S. Hopyan, "Unicameral Bone Cysts: comparison of percutaneous curettage, steroid, and autologous bone marrow injections," Journal of Pediatric Orthopaedics, vol. 31, no. 1, pp. 50-55, 2011.

[21] J. M. Newquist, G. M. Baxter, R. Sangeeta, and F. Olea-Popelka, "Evaluation of plasma fibrinogen concentration as an indicator of physeal or epiphyseal osteomyelitis in foals: 17 cases (20022007)," Journal of the American Veterinary Medical Association, vol. 235, no. 4, pp. 415-419, 2009. 

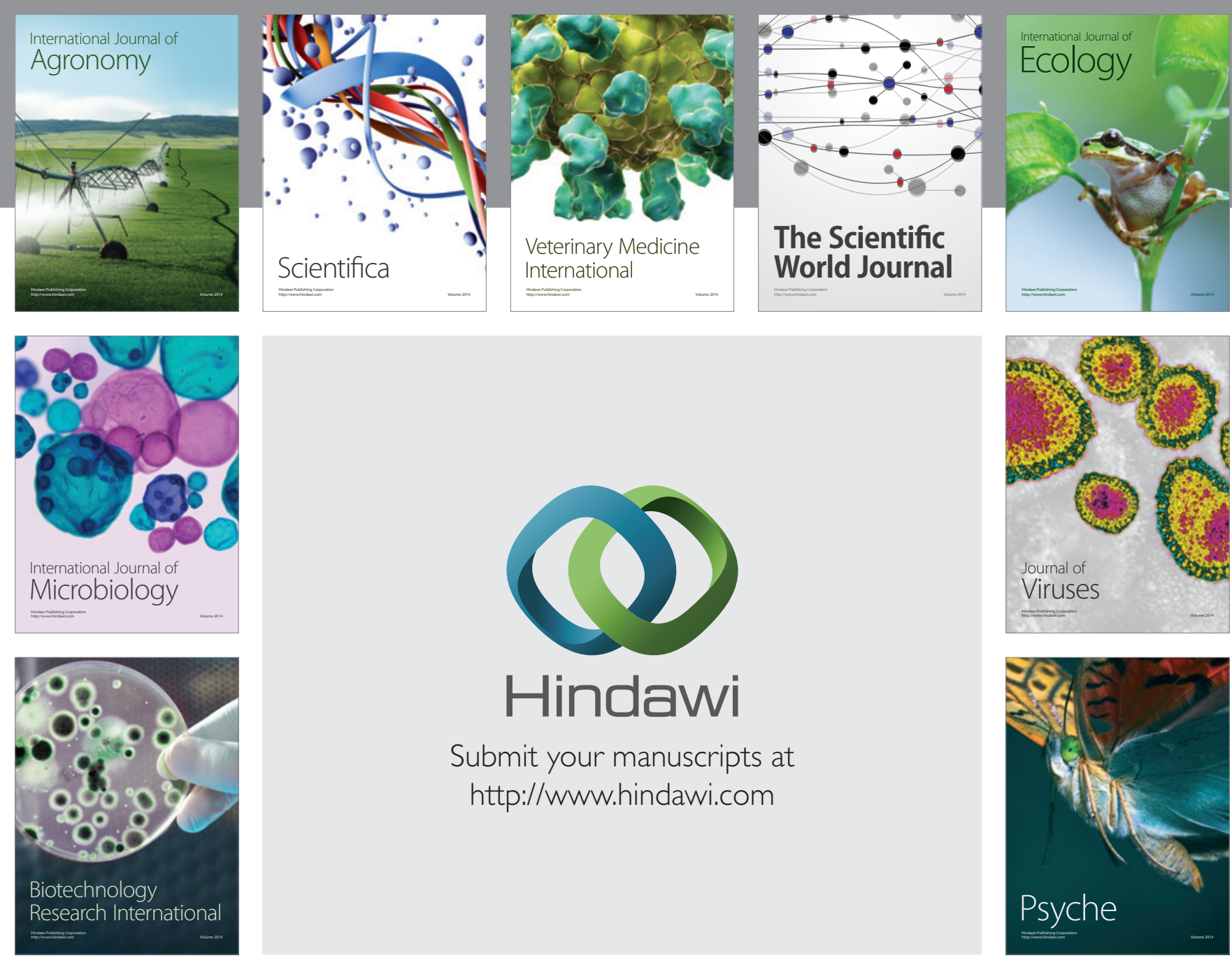

Submit your manuscripts at http://www.hindawi.com
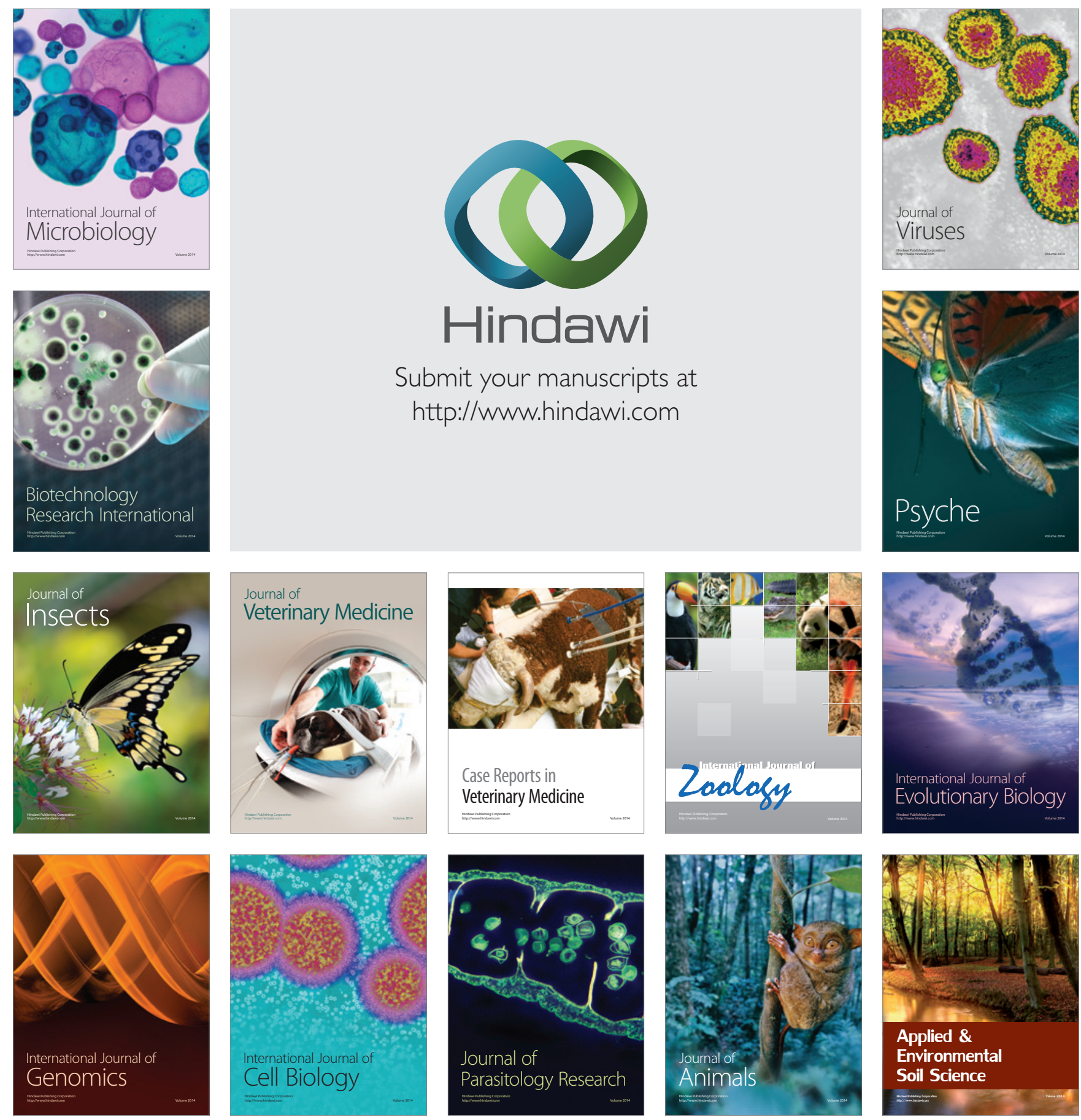